\title{
Endoskopie des peripheren Lungenrundherdes
}

\author{
Endoscopy of Peripheral Lung Nodule
}

Autoren

Institut
R. Eberhardt, D. Gompelmann, F. J. F. Herth

Abteilung Pneumologie und Beatmungsmedizin, Thoraxklinik am Universitätsklinikum Heidelberg (Chefarzt Prof. Dr. med. Felix J. F. Herth) eingereicht $\quad 29.8 .2011$ akzeptiert nach Revision 14. 9. 2011

\section{Bibliografie}

Dol http://dx.doi.org/ 10.1055/s-0031-1291399 Online-Publikation: 10.11.2011 Pneumologie 2011; 65: 745-750 (c) Georg Thieme Verlag KG Stuttgart · New York ISSN 0934-8387

\section{Korrespondenzadresse} PD Dr. med. Ralf Eberhardt Abteilung Pneumologie und Beatmungsmedizin Thoraxklinik am Universitätsklinikum Heidelberg Amalienstraße 5 69126 Heidelberg ralf.eberhardt@thoraxklinikheidelberg.de www.thoraxklinik-heidelberg.de

Serienherausgeber A. Valipour, Wien

\section{Zusammenfassung \\ $\nabla$}

Lungenrundherde unklarer Dignität, häufig zufallsbefundlich entdeckt, stellen weiterhin eine diagnostische Herausforderung dar. Neben der operativen Resektion stehen die transthorakale Punktion und die Bronchoskopie mit transbronchialer Biopsie als minimal-invasive Verfahren zur Verfügung. Durch den Einsatz neuer Navigationstechniken lässt sich die Aussagekraft der Bronchoskopie zur Diagnostik von Lungenrundherden gegenüber der Röntgendurchleuchtung deutlich verbessern. Dabei ist ein Einsatz von endobronchialem Ultraschall, elektromagnetischer Navigation und virtueller Bronchoskopie auch ohne Röntgendurchleuchtung möglich und ihre Kombination steigert die Sensitivität. Diese Verfahren haben bereits einen festen Stellenwert bei Patienten mit einem unklaren peripheren Lungenbefund, bei denen aufgrund eines hohen operativen Risikos ein primärer chirurgischer Eingriff nicht möglich und bei denen eine Diagnose für die weitere Therapieplanung notwendig ist. Möglicherweise werden durch diese Techniken zukünftig aber auch erste endoskopische Therapieansätze möglich.

\section{Einleitung \\ $\nabla$}

Rund 32000 Männer und 13000 Frauen erkranken jährlich in Deutschland an Lungenkrebs. Obwohl die Risikofaktoren sehr gut bekannt sind, wird das Bronchialkarzinom meist erst in einem fortgeschrittenen Stadium diagnostiziert. Epidemiologische Daten belegen dabei eine strenge Abhängigkeit der Prognose vom Stadium der Erkrankung, sodass Patienten mit Lungenkrebs im Vergleich zu anderen Karzinomen die ungünstigste Prognose haben [1 - 2].

Der Wunsch nach Früherkennung des Bronchialkarzinoms hat in den vergangenen Jahren $\mathrm{zu}$ einem vermehrten Einsatz der Computertomo-

\section{Abstract \\ $\nabla$}

Pulmonary nodules of unknown dignity, often incidental findings, still present a diagnostic challenge. Next to operative resection, we find minimal invasive therapies such as transthoracal punctions and bronchoscopy with transbronchial as further options. By using modern navigation techniques, the validity of bronchoscopy in diagnosing pulmonary nodules, compared to x-ray fluoroscopy, has already been improved. Thus, the use of endobronchial ultrasound, electromagnetic navigation and virtual bronchoscopy - even without x-ray fluoroscopy - is possible and the combination augments sensitivity. These techniques already rate high in patients with unclear peripheral pulmonary nodules and high risk profiles to surgical operations, thus not to be operated, but still in need of further diagnostic procedures in order to plan therapy options. A future use of endoscopic therapies may possibly be a first approach.

grafie (CT) geführt, obwohl in der Vergangenheit weder für die Sputumanalyse noch für ein radiologisches Screening-Verfahren ein verbessertes Überleben von Risikopatienten nachgewiesen werden konnte [3 -5]. In dem kürzlich veröffentlichten amerikanischen National Lung Screening Trial (NLST) mit mehr als 53000 Probanden konnte erstmals gezeigt werden, dass eine Senkung der Mortalität durch die Anwendung der SpiralCT als Screeningmethode bei einer bestimmten Patientenpopulation möglich ist. So konnte mit einem dreimaligen Computertomografiescreening im Vergleich zur konventionellen Röntgenaufnahme bei schweren Rauchern oder Ex-Rauchern im Alter zwischen 55 und 74 Jahren die 
durch Lungenkrebs bedingte Sterblichkeit um 20,0\% im Vergleich zu der randomisierten Kontrollgruppe gesenkt werden [6].

Durch die zunehmende Durchführung von computertomografischen Untersuchungen des Thorax, auch aus anderen Indikationen, ist eine ansteigende Zahl von zufallsbefundlich entdeckten, insbesondere kleineren Lungenrundherden zu erwarten [7-8]. Es ist davon auszugehen, dass bei asymptomatischen aktiven oder ehemaligen Rauchern in 20 - 50\% der Fälle ein oder mehrere nicht-verkalkte Rundherde in der CT der Lunge zu finden sind [9 -10]. Eine frühzeitige diagnostische Klärung aufgrund ihrer möglichen malignen Dignität bleibt dabei für die Prognose entscheidend.

\section{Der periphere Lungenrundherd \\ $\nabla$}

Ein solitärer Lungenrundherd ist definiert als eine singuläre, runde bzw. umschriebene Läsion mit vermehrter Dichte, die kleiner als $3 \mathrm{~cm}$ und von normalem Lungengewebe umgeben ist. Radiologisch bleibt er ohne Nachweis einer zusätzlichen Lymphknotenmetastasierung, einer nachgeschalteten Atelektase oder eines Pleuraergusses [11]. Typischerweise ist der Lungenrundherd endobronchial nicht sichtbar und entzieht sich somit der einfachen diagnostischen Biopsie unter visueller Kontrolle.

Die Wahrscheinlichkeit für eine maligne Genese der zufallsbefundlich in der CT entdeckten pulmonalen Rundherde ist insbesondere von der Größe bzw. ihrem Durchmesser abhängig. Da die Karzinomprävalenz bei Patienten mit Lungenrundherden bis $8 \mathrm{~mm}$ und fehlender Tumorerkrankung in der Anamnese niedrig ist und die bioptischen Möglichkeiten schwierig sind, wird in den nationalen und internationalen Leitlinien zunächst eine radiologische Verlaufskontrolle empfohlen $[1,7]$. Das einzige Kriterium für Benignität eines Rundherdes ist dabei die Größenstabilität, wobei die zum Teil langen Tumorverdopplungszeiten berücksichtigt werden müssen, sodass auch eine radiologische Stabilität von mehr als 2 Jahren eine maligne Genese nicht vollständig ausschließt [12].

Bereits bei einer Größe von mehr als $8 \mathrm{~mm}$ im Durchmesser steigt die Malignomwahrscheinlichkeit jedoch so stark an, dass eine radiologische Verlaufskontrolle nicht mehr ausreichend ist.

\section{Nicht-endoskopische Diagnostik des Lungenrundherdes \\ $\nabla$}

Bei hoher Prätestwahrscheinlichkeit für ein Malignom stellt die chirurgische Resektion den Goldstandard mit der höchsten Sensitivität und Spezifität dar, wobei der Eingriff nicht nur diagnostisch, sondern bereits therapeutisch sein kann. Eine vorherige Biopsie ist bei funktioneller Operabilität in der Regel nicht notwendig.

Bei eingeschränkter oder fehlender Operabilität oder in Fällen mit geringer bis mittlerer Wahrscheinlichkeit für eine maligne Erkrankung kann eine Positronenemissionstomografie (PET) mit Fluoro-2-desoxy-D-Glucose (FDG) durchgeführt werden, da maligne Läsionen einen erhöhten Glucosemetabolismus und somit eine erhöhte Aufnahme von FDG aufweisen [13-14]. Mit falsch negativen Befunden ist insbesondere bei gut differenzierten Tumoren wie dem lepidischen Karzinom und dem typischen Karzinoid zu rechnen, während falsch positive Befunde insbesondere bei granulomatösen Entzündungen wie bei Tuberkulose und Sarkoidose, aber auch bei bakteriellen oder mykotischen Infektionen zu erwarten sind. Auch die Größe des pulmonalen Rundherdes beeinflusst die diagnostische Aussagefähigkeit der PET mit häufig falsch-negativen Befunden bei Herden unter 7-10 mm.

Die eingeschränkte Operabilität aufgrund der Komorbiditäten der Patienten stellt die häufigste Indikation für einen Versuch zur minimal-invasiven Diagnostik dar [15]. In einer retrospektiven Analyse an 1432 Patienten, die zur Resektion eines solitären Lungenrundherdes zugewiesen wurden, waren entsprechend nur 27\% geeignete Kandidaten für einen chirurgischen Eingriff. Die Patienten hatten in mehr als der Hälfte eine Kontraindikation für eine Operation oder wiesen ein hohes Risiko für eine perioder postoperative Komplikation auf [16].

Durch eine transthorakale Punktion können pulmonale Läsionen sowohl zytologisch als auch histologisch diagnostiziert werden. Bei nicht pleuraständigen Lungenherden kommt dabei heutzutage in der Regel die Computertomografie zum Einsatz. Ihre Sensitivität für Malignität wird in der Literatur mit $74-96 \%$ bei einer Spezifität von annähernd $100 \%$ angegeben, ist aber von der Größe der Läsion und ihrer Lokalisation bzw. dem Abstand zur Pleura abhängig. Die häufigsten Komplikationen sind dabei ein postinterventioneller Pneumothorax mit 15-44\% bzw. eine pulmonale Blutung [17-18].

\section{Diagnostische Endoskopie \\ $\nabla$}

Das endoskopische Standardverfahren in der Diagnostik pulmonaler Rundherde ist die Bronchoskopie mit transbronchialer Biopsie (TBB) unter gleichzeitiger Röntgendurchleuchtung. Eine TBB kann auch unter Thrombozytenaggregationshemmung mit Acetylsalicylsäure durchgeführt werden, während eine Gerinnungshemmung mit Clopidogrel aufgrund der erhöhten Blutungsgefahr im Vorfeld abgesetzt werden sollte [19-20]. Komplikationen bei der Bronchoskopie mit TBB sind selten und die Pneumothoraxrate wird in der Literatur mit $<3 \%$ angeben [21]. Dabei variiert die diagnostische Wertigkeit dieser Methode je nach Größe und Lage der pulmonalen Läsion, ihrem Verhältnis zu den Atemwegen sowie der Darstellbarkeit unter Röntgendurchleuchtung stark [22]. Für periphere pulmonale Läsionen zwischen $2,5 \mathrm{~cm}$ und $4,0 \mathrm{~cm}$ wird eine bronchoskopische Trefferquote unter Röntgenkontrolle von $62 \%$ beschrieben, während die Sensitivität bei Patienten mit tumorverdächtigen Rundherden $<2,5 \mathrm{~cm}$ in der gleichen Arbeit bereits unter $40 \%$ lag [7]. Für Läsionen $<20 \mathrm{~mm}$ wurde in einer Metaanalyse von Schreiber et al. sogar nur eine Sensitivität von 33\% gefunden [4].

Durch den Einsatz weiterer Biopsieinstrumente lässt sich die diagnostische Aussagekraft der Bronchoskopie in der Diagnostik von peripheren pulmonalen Läsionen verbessern. Insbesondere für die Kombination der Zangenbiopsie mit der transbronchialen Nadelaspiration (TBNA) konnte in zahlreichen Arbeiten der diagnostische Zugewinn durch das additive Biopsieverfahren bereits unter Röntgendurchleuchtung gezeigt werden [23-24]. Durch die Methoden der Katheteraspiration lässt sich ebenfalls die diagnostische Trefferquote verbessern [25]. Die zytologischen Verfahren sind dabei stark von der Erfahrung und den Fertigkeiten des untersuchenden Zytologen abhängig, aber die Anzahl falsch-positiver zytologischer Befunde wird in der Literatur als sehr gering angesehen $(<0,4 \%)(\bullet$ Tab. 1$)$.

Eine Steuerung der Bronchoskopie und der Biopsiezange mittels Computertomografie verbessert zwar die Bildgebung, aber im Vergleich zur Röntgendurchleuchtung nicht die Trefferquote oder die Komplikationsrate. Zudem limitieren Strahlenbelastung 
für Patient und Untersucher sowie die Untersuchungsdauer in der CT den Nutzen für die tägliche Routineanwendung [26 - 27]. Die Vorteile der Bronchoskopie mit der transbronchialen Gewebeentnahme sind gegenüber der transthorakalen Punktion ihre geringe Invasivität bzw. ihre niedrige Komplikationsrate, jedoch bedarf es Navigationstechniken, um ihre Sensitivität zu erhöhen.

\section{Peripherer endobronchialer Ultraschall}

\section{$\nabla$}

Ultraschall wird an der Grenzschicht zur Luft vollständig reflektiert, trotzdem kann er zur Diagnostik peripherer Rundherde eingesetzt werden. Radiale Ultraschallsonden, sogenannte Miniprobes, können über den Instrumentierkanal eines herkömmlichen flexiblen Bronchoskops in die Lungenperipherie vorgeschoben werden. In normalem und somit ventiliertem Lungenparenchym wird durch die Totalreflexion ein „schneesturmartiges Bild“ dargestellt, während pulmonale Raumforderungen, die mit einer Schallabsorption einhergehen, gut differenziert werden können. Somit lassen sich intrapulmonale Rundherde mittels endobronchialem Ultraschall (EBUS) detektieren [28] ( $\bullet$ Abb.1). Der radiale EBUS kann als alleinige Technik oder in Kombination mit einer Röntgendurchleuchtung eingesetzt werden. Bei der Kathetertechnik wird die Ultraschallsonde in einem dünnen Katheter vorgeführt, diese nach Erreichen des Lungenrundherdes entfernt und der Katheter als verlängerter Arbeitskanal vor Ort belassen. Somit können Biopsieinstrumente zielgerichtet zu den Läsionen vorgeschoben werden, wodurch eine zusätzliche Röntgendurchleuchtung entbehrlich wird bzw. die Methode auch bei Lungenrundherden, die radiologisch nicht sichtbar sind, erfolgreich angewendet werden kann [29-30].

Die Erfolgsquote der EBUS-gesteuerten TBB hängt dabei von der Position des Biopsiekatheters in Relation zur pulmonalen Raumforderung ab. So konnten Yamada et al. zeigen, dass bei Läsionen, bei denen die EBUS-Sonde in der Läsion platziert werden konnte,

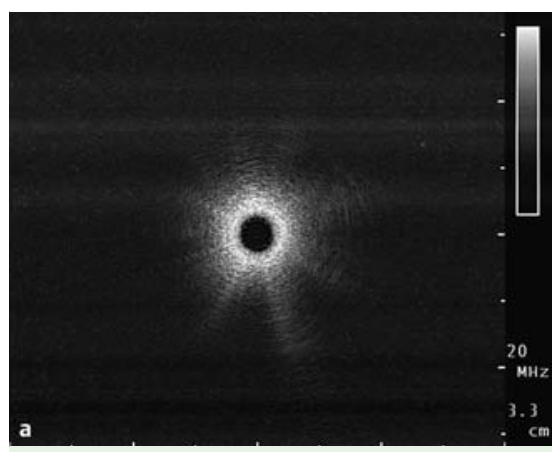

Abb. 1 a Das Ultraschallbild zeigt das typische „Schneegestöber“ bei normalem Lungengewebe, welches einer kompletten Reflexion des Ultraschalls an der Grenze zur umliegenden ventilierten Lunge entspricht.

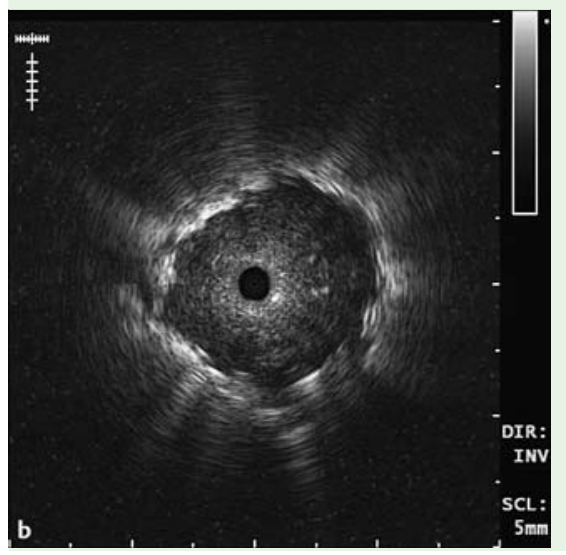

b Plattenepithelkarzinom der Lunge im rechten Unterlappen mit einem Durchmesser von $15 \mathrm{~mm}$. Das Ultraschallbild zeigt einen soliden Tumor, der sich deutlich zum umliegenden Alveolargewebe abgrenzt Radiale Ultraschallsonde, sog. Miniprobe Olympus, Tokio, Japan) die Erfolgsaussichten auf eine definitive Diagnose signifikant höher waren, als wenn sie nur randständig erreicht werden konnten oder gar nicht darstellbar waren ( $83 \%$ vs. $61 \%$ vs. $4 \%$ ) [31]. Die additive Durchführung von transbronchialen Nadelaspirationen führt aber bei peripheren Läsionen, bei denen die EBUS-Sonde nur tangential platziert werden kann, zu einer signifikanten Verbesserung des sog. Diagnostic Yield, während bei einem möglichen Vorschieben zentral in den Lungenherd sich keine Unterschiede zwischen transbronchialer Zangenbiopsie und TBNA ergeben [32].

Insgesamt konnte in zahlreichen Arbeiten bestätigt werden, dass durch den Einsatz von radialen EBUS-Sonden die diagnostische Trefferquote der Bronchoskopie mit transbronchialer Biopsie bei Lungenrundherden erhöht werden kann. Die veröffentlichten Ergebnisse sind besser als die für die konventionelle TBB unter alleiniger Röntgenkontrolle. In einer kürzlich publizierten Metanalyse für die EBUS-gesteuerte Diagnostik von peripheren pulmonalen Läsionen wurden 16 Studien mit mehr als 1400 Patienten eingeschlossen. Aus letztendlich 13 Arbeiten konnten Steinfort et al. eine gepoolte Sensitivität von $0,73(0,70-0,76)$ bei einer Spezifität von 1,00 (0,99-1,00) für die Diagnostik eines Bronchialkarzinoms bzw. pulmonalen Malignoms errechnen [33]. Bei Rundherden kleiner $20 \mathrm{~mm}$ liegt die Trefferquote mit 46\% deutlich niedriger. Lassen sich die kleinen pulmonalen Läsionen jedoch mittels Ultraschall detektieren, so kann in mehr als zwei Drittel der Fälle mit einer definitiven Diagnose gerechnet werden [34].

\section{Elektromagnetische Navigation} $\nabla$

Einen anderen Ansatz in der endoskopischen Diagnostik von pulmonalen Rundherden stellt die elektromagnetische Navigation (EN) dar. Sie ermöglicht eine bildgestützte Steuerung des Bronchoskops und besteht aus vier verschiedenen Komponenten [35]. Mithilfe eines sog. Electromagnetic Board wird ein niederfrequentes, elektromagnetisches Feld um den Thorax des zu untersuchenden Patienten generiert. Durch das elektromagnetische Feld lässt sich die Position eines kleinen Sensors an der Spitze eines Katheters, der durch den Arbeitskanals eines flexiblen Bronchoskops eingeführt wird, im Patienten relativ exakt bestimmen. Dabei können sowohl die Ausrichtung als auch Rotationsbewegungen des Sensors erfasst werden. Durch den Steuerungsmechanismus am Handgriff des Katheters lässt sich dabei die Spitze in acht verschiedene Richtungen abwinkeln. Damit wird das Steuern im peripheren Bronchialsystem und somit ein zielgerichtetes Vorführen des Kathetersystems in verschiedene Atemwege möglich. Durch Überlagerung rekonstruierter CT-Daten mit der Lage der Katheters im elektromagnetischen Feld lässt sich der Katheter zielgerichtet auf einen zuvor markierten Punkt vorführen und somit pulmonale Rundherde ansteuern. Nach maximaler Annäherung an die periphere Läsion wird dann der sog. Locatable Guide (LG) entfernt und der vor Ort als verlängerter Arbeitskanal belassene Katheter als Leitschiene für Biopsieinstrumente genutzt. Ein erneutes Einführen des Sensors nach Abschluss der Biopsieentnahme zeigt eine eventuelle Katheterdislokation an (৫ Abb. 2).

In den ersten Arbeiten zur EN konnte nachgewiesen werden, dass pulmonale Läsionen unter zusätzlicher Verwendung einer Röntgendurchleuchtung mit diesem Navigationssystem diagnostiziert werden können. Gildea et al. erreichten dabei eine Trefferquote von $74 \%$, wobei mehr als die Hälfte der Rundherde weniger als 


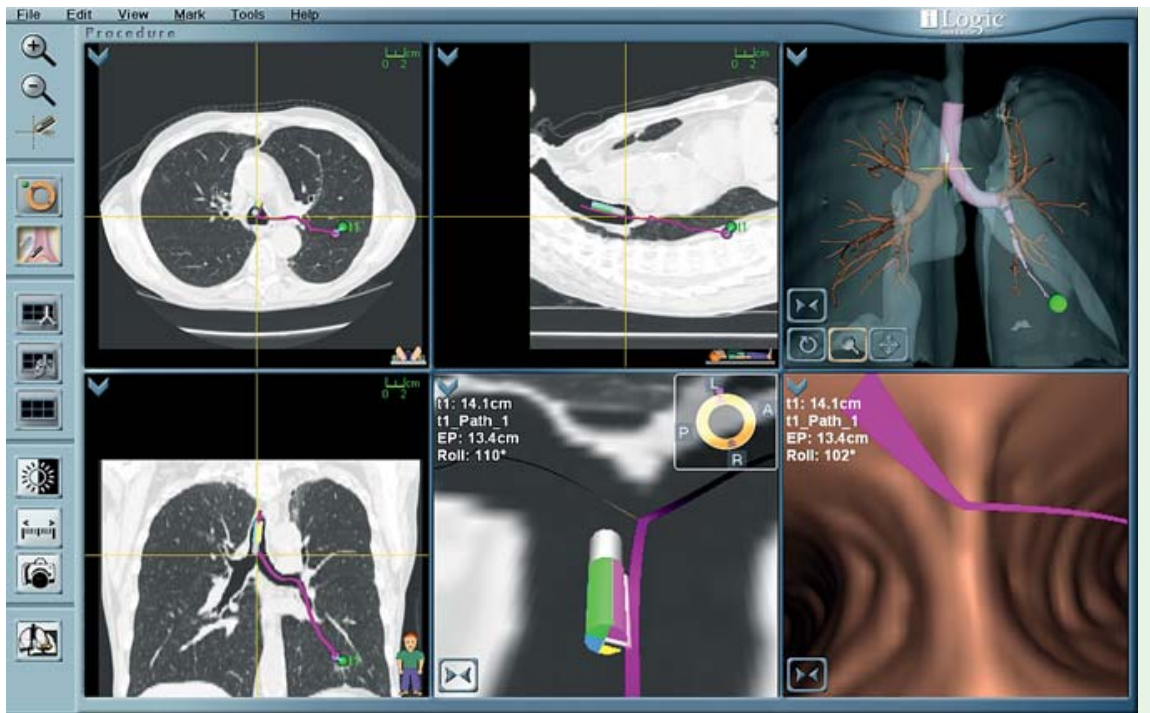

Abb. 2 Computersystem InReach ${ }^{\mathrm{TM}}$ (superDimension Inc., Minneapolis, MN, USA). Auf dem Bildschirm wird die Position des Sensors in drei Schnittebenen in Echtzeit angezeigt. Der zu folgende Bronchialbaum (lila) ist rechts oben dargestellt. Die virtuelle Bronchoskopie ist in dieser Softwareversion bereits integriert.

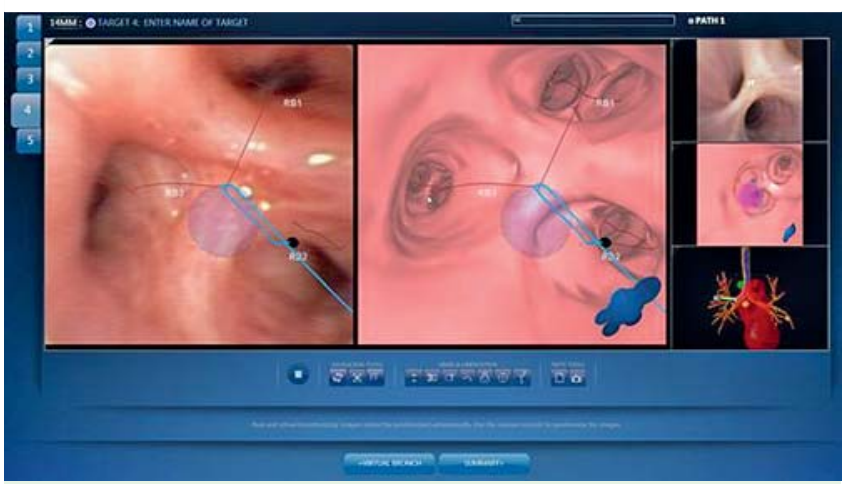

Abb. 3 LungPoint Virtual Bronchoscopic Navigation System (Broncus Technologies Inc., Mountain View, CA, USA). Im Untersuchungsmodus wird die errechnete Route im realen Bronchoskopiebild blau angezeigt. Der Pfad führt den Untersucher zu einem Lungenrundherd im dorsalen Oberlappen rechts.

$2 \mathrm{~cm}$ betrug [36]. Die Autoren fanden dabei keinen signifikanten Unterschied in ihrer Erfolgsrate in Abhängigkeit von der Herdgröße.

Zwei weitere Arbeiten untersuchten die Technik der EN ohne den zusätzlichen Einsatz der Röntgendurchleuchtung [35, 37]. Beide Arbeitsgruppen konnten bestätigen, dass mit dem Einsatz der virtuellen Navigationstechnik allein nicht nur eine vergleichbare Trefferquote wie mit zusätzlicher Röntgendurchleuchtung zu erzielen ist, sondern auch zu keinem Anstieg der Komplikationsrate führt.

Durch die Kombination von elektromagnetischer Navigation und radialem, endobronchialem Ultraschall lässt sich die diagnostische Erfolgsrate bei peripheren pulmonalen Läsionen auf bis zu $88 \%$ verbessern [38]. Während für den EBUS die Trefferquote in den Oberlappen aufgrund der Abwinklung und der damit schwierigeren Steuerung der Ultraschallsonden tendenziell schlechter ist, beeinflussen die Atembewegungen bzw. die unterschiedlichen Inspirationstiefen in der CT und bei der Bronchoskopie die Ergebnisse der EN bei Rundherden in den Unterlappen negativ. Durch die Vereinigung der Vorteile der jeweiligen Navigationsverfahren, der präzisen Navigation durch den LG sowie der visuellen Bestätigung des Rundherdes in Echtzeit durch den EBUS lassen sich die Limitationen der jeweiligen Untersuchungs- techniken ausgleichen. Dabei ist ebenfalls eine zusätzliche Anwendung eines zytologischen Verfahrens bei der EN zu empfehlen, zumindest wenn die EBUS-Kontrolle ein Nichterreichen des Lungenrundherdes anzeigt oder eine radiale Ultraschallkontrolle nicht verfügbar ist [34].

\section{Virtuelle Bronchoskopie \\ $\nabla$}

Ultradünne Bronchoskope mit einem äußeren Durchmesser von weniger als $3 \mathrm{~mm}$ stehen seit einigen Jahren für den klinischen Einsatz zur Verfügung und können wesentlich weiter in die peripheren Atemwege vorgeschoben werden als herkömmliche Bronchoskope. Die Variabilität der Bronchien nimmt aber mit zunehmender Aufteilung stark zu und stellt insbesondere für die Orientierung in den peripheren Atemwegen eine entscheidende Herausforderung für den Untersucher dar.

Anhand von CT-Daten können die Atemwege computergestützt rekonstruiert und als virtuelle Bronchoskopie (VB) dargestellt werden. Softwaresysteme ermöglichen nun, einen Pfad zu einer peripheren Läsion im Vorfeld einer diagnostischen Bronchoskopie zu planen. Die Auflösung der VB und die Länge des rekonstruierten Bronchialbaumes sind aber sehr stark von der Schichtdicke und der Qualität der verwendeten Computertomografie abhängig.

Ein neues Navigationssystem zeigt im Untersuchungsmodus das endoskopische Bild neben der virtuellen Bronchoskopie. Über einen Synchronisationsmodus wird dann der Pfad in das Untersuchungsbild eingefügt ( $\mathbf{A}$ bb. $\mathbf{3}$ ). In einer ersten Machbarkeitsstudie konnten mit diesem System ohne weitere Navigationstechnik 20 von 25 der peripheren Lungenherde (mittlerer Durchmesser $28 \pm 0,7 \mathrm{~mm}$ ) diagnostiziert werden [39]. Eine zusätzliche Anwendung des peripheren EBUS ist durch die Entwicklung von ultradünnen Sonden bereits ebenfalls möglich. So erreichten Asahina et al. mit der Kombination eine Sensitivität von 44,4\% für Läsionen $<20 \mathrm{~mm}$ und 91,7\% für Lungenrundherde von 20 $30 \mathrm{~mm}$ [40]. Auch in der neuesten EN-Software wurde eine zusätzliche virtuelle Bronchoskopie bereits implementiert.

Trotz zunehmend miniaturisierter Videotechnik sind bei der Verwendung von ultradünnen Bronchoskopen mit immer kleinerem Außendurchmesser die Größe des Arbeitskanals und somit auch der Biopsieinstrumente begrenzt. Als Alternative zu kleinsten 
Tab. 1 Bronchoskopische Biopsietechniken bei peripheren Lungenrundherden.

\begin{tabular}{|ll|}
\hline Biopsietechnik & \\
\hline Zangenbiopsie & Standardverfahren \\
\hline $\begin{array}{l}\text { Transbronchiale Nadelaspiration } \\
\text { (TBNA) }\end{array}$ & diagnostischer Zugewinn \\
\hline Bürstenzytologie & ohne diagnostischen Zugewinn \\
\hline Katheteraspiration & diagnostischer Zugewinn \\
\hline Kryobiopsie & noch experimentell \\
\hline
\end{tabular}

Tab. 2 Bronchoskopische Navigationstechniken bei pulmonalen Rundherden $<3 \mathrm{~cm}$.

\begin{tabular}{|c|c|c|}
\hline Navigationstechnik & Sensitivität & Bewertung \\
\hline Röntgendurchleuchtung & $40 \%$ & Standardverfahren \\
\hline $\begin{array}{l}\text { CT-gesteuerte transbron- } \\
\text { chiale Biopsie (TBB) }\end{array}$ & $70 \%$ & $\begin{array}{l}\text { Strahlenbelastung } \\
\text { logistisch aufwendig }\end{array}$ \\
\hline $\begin{array}{l}\text { Peripherer endobronchialer } \\
\text { Ultraschall (EBUS) }\end{array}$ & $70 \%$ & $\begin{array}{l}\text { einfache Anwendung } \\
\text { kein real-time-Verfahren }\end{array}$ \\
\hline $\begin{array}{l}\text { Elektromagnetische } \\
\text { Navigation (EN) }\end{array}$ & $70 \%$ & $\begin{array}{l}\text { gute Steuerbarkeit } \\
\text { Mehrkosten }\end{array}$ \\
\hline $\begin{array}{l}\text { Virtuelle Bronchoskopie } \\
\text { (VB) }\end{array}$ & $80 \% *$ & $\begin{array}{l}\text { von der CT-Qualität } \\
\text { abhängig } \\
\text { kleine Biopsien, nur } \\
\text { wenig Daten }\end{array}$ \\
\hline
\end{tabular}

* bei pulmonalen Läsionen bis $40 \mathrm{~mm}$

Zangenbiopsien müssen daher zukünftig auch zytologische Verfahren oder Kältesonden [41] zur Probenentnahme geprüft werden.

\section{Therapeutische Endoskopie}

Durch den Einsatz von Navigationstechniken lässt sich die bronchoskopische Diagnostik von peripheren Lungenrundherden im Vergleich zur konventionellen transbronchialen Biopsie unter Röntgendurchleuchtung verbessern ( Tab.2). Ihr Einsatz in der klinischen Routine muss aber aufgrund des technischen Aufwandes und der zusätzlichen Kosten jedoch stets kritisch hinterfragt werden. Dennoch haben die Verfahren derzeit bereits einen hohen Stellenwert bei Patienten mit einem unklaren peripheren Lungenbefund und einem hohen operativen Risiko, bei denen eine Diagnose für die weitere Therapieplanung notwendig ist.

Die Zukunft dieser Navigationsverfahren ist jedoch in der minimal-invasiven, bronchoskopischen Therapie von Lungenrundherden zu sehen. Erste Therapieansätze befinden sich bereits in Erprobung. So werden mithilfe der EN oder des EBUS sog. Fiducials zur Steuerung einer externen Bestrahlung von inoperablen Lungentumoren eingesetzt [42-44]. Auch beschrieben Harms et al. die Möglichkeit der endoluminalen Brachytherapie von peripheren Lungentumoren. Die Einlage des Strahlenkatheters erfolgte mittels EN, kombiniert mit einer EBUS-Kontrolle der Sondenlage. Nach nasaler Ausleitung konnte dann der Tumor mit einer HDRBrachytherapie mit Iridium ${ }^{192}$ im Afterloading-Verfahren bestrahlt werden. In der Schonung des umliegenden Lungengewebes und der Möglichkeit, das bronchovaskuläre Bündel und somit die Lymphabflusswege mitzubestrahlen, sehen die Autoren mögliche Vorteile gegenüber einer externen kleinvolumigen Bestrahlung [45].
Die Methode mit dem größten Zukunftspotential stellt aber sicherlich die Radiofrequenzablation (RFA) dar. Bei der RFA wird eine Applikationssonde in das Gewebe eingebracht und eine Koagulationsnekrose durch die Wärmeentwicklung des Hochfrequenzstroms erzeugt. Die Radiofrequenzablation wird bereits auch transthorakal bei pulmonalen Malignomen erfolgreich eingesetzt. In einer prospektiven, multizentrischen Studie mit mehr als 100 Patienten konnten mit dieser CT-gesteuerten Technik in $88 \%$ eine lokale komplette Remission und ein 1-Jahresüberleben von $70 \%$ erzielt werden. Allerdings war hier die Komplikationsund Pneumothoraxrate deutlich erhöht [46]. Eine japanische Arbeitsgruppe veröffentlichte 2010 ihre ersten Erfahrungen über eine bronchoskopische RFA bei zehn Patienten mit einem peripheren, nicht-kleinzelligen Bronchialkarzinom. Die Autoren hatten bereits tierexperimentell nachweisen können, dass zur Vermeidung eines schnellen Anstiegs der Impedanz gekühlte Sonden notwendig sind. In der histologischen Aufarbeitung der anschließend resezierten Bronchialkarzinome zeigte sich eine noch unzureichende Größe des Ablationsareals, sodass für diesen Therapieansatz sicherlich noch zahlreiche technische Weiterentwicklungen notwendig sind [47-48]. Trotzdem erscheint nun das Ziel, unklare Lungenrundherde in einer bronchoskopischen Untersuchung zu diagnostizieren und anschließend in gleicher Sitzung kurativ zu behandeln, denkbar.

\section{Interessenkonflikt}

\section{$\nabla$}

Die Autoren geben an, dass kein Interessenkonflikt besteht.

\section{Literatur}

1 Vogelmeier C, Buhl R, Criee CP et al. [Guidelines for the diagnosis and therapy of COPD issued by Deutsche Atemwegsliga and Deutsche Gesellschaft für Pneumologie und Beatmungsmedizin]. Pneumologie 2007; 61: $1-40$

2 Jemal A, Siegel $R$, Ward $E$ et al. Cancer statistics, 2008. CA Cancer J Clin 2008; 58: $71-96$

3 Fontana RS, Sanderson DR, Taylor WF et al. Early lung cancer detection: results of the initial (prevalence) radiologic and cytologic screening in the Mayo Clinic study. Am Rev Respir Dis 1984; 130: 561 - 565

4 Schreiber G, McCrory DC. Performance characteristics of different modalities for diagnosis of suspected lung cancer: summary of published evidence. Chest 2003; 123: $115-128$

5 Infante M, Cavuto S, Lutman FR et al. A randomized study of lung cancer screening with spiral computed tomography: three-year results from the DANTE trial. Am J Respir Crit Care Med 2009; 180: 445 - 453

6 Aberle DR, Adams AM, Berg CD et al. Reduced lung-cancer mortality with low-dose computed tomographic screening. N Engl J Med 2011; 365: $395-409$

7 Gould MK, Fletcher J, Iannettoni MD et al. Evaluation of patients with pulmonary nodules: when is it lung cancer? ACCP evidence-based clinical practice guidelines (2nd edition) Chest 2007; 132: 108-130

8 Midthun DE. Caution: screening for lung cancer. Expert Rev Respir Med 2009; 3: 203-205

9 Henschke CI, McCauley DI, Yankelevitz DF et al. Early Lung Cancer Action Project: overall design and findings from baseline screening. Lancet 1999; 354: $99-105$

10 Swensen SJ, Jett JR, Hartman TE et al. Lung cancer screening with CT: Mayo Clinic experience. Radiology 2003; 226: 756-761

11 Tuddenham WJ. Glossary of terms for thoracic radiology: recommendations of the Nomenclature Committee of the Fleischner Society. AJR Am J Roentgenol 1984; 143: 509-517

12 Hartman TE. Radiologic evaluation of the solitary pulmonary nodule. Radiol Clin North Am 2005; 43: 459-465

13 Pastorino $U$, Bellomi $M$, Landoni $C$ et al. Early lung-cancer detection with spiral CT and positron emission tomography in heavy smokers: 2-year results. Lancet 2003; 362: $593-597$

14 Ung YC, Maziak DE, Vanderveen JA et al. 18Fluorodeoxyglucose positron emission tomography in the diagnosis and staging of lung cancer: a systematic review. J Natl Cancer Inst 2007; 99: 1753-1767 
15 Bolliger $C T$. Functional reserve before lung resection: how low can we go? Respiration 2009; 78: 20-22

16 Gasparini S. Solitary pulmonary nodule. In: Strausz J, Bolliger CT eds. Interventional Pulmonology. 48. Plymouth: Latimer Trend \& Co; 2010: $90-108$

17 Ohno Y, Hatabu H, Takenaka D et al. CT-guided transthoracic needle aspiration biopsy of small ( $<$ or $=20 \mathrm{~mm}$ ) solitary pulmonary nodules. AJR Am J Roentgenol 2003; 180: 1665 - 1669

18 Priola AM, Priola SM, Cataldi A et al. Accuracy of CT-guided transthoracic needle biopsy of lung lesions: factors affecting diagnostic yield. Radiol Med 2007; 112: 1142 - 1159

19 Herth FJ, Becker HD, Ernst A. Aspirin does not increase bleeding complications after transbronchial biopsy. Chest 2002; 122: 1461 - 1464

20 Ernst A, Eberhardt R, Wahidi M et al. Effect of routine clopidogrel use on bleeding complications after transbronchial biopsy in humans. Chest 2006; 129: 734-737

21 Izbicki G, Shitrit D, Yarmolovsky A et al. Is routine chest radiography after transbronchial biopsy necessary? A prospective study of 350 cases. Chest 2006; 129: 1561 - 1564

22 Ost $D$, Fein AM, Feinsilver SH. Clinical practice. The solitary pulmonary nodule. N Engl J Med. 2003; 348: 2535 - 2542

23 Gasparini S, Ferretti $M$, Secchi $E B$ et al. Integration of transbronchial and percutaneous approach in the diagnosis of peripheral pulmonary nodules or masses. Experience with 1,027 consecutive cases. Chest 1995; 108: $131-137$

24 Reichenberger $F$, Weber J, Tamm $M$ et al. The value of transbronchial needle aspiration in the diagnosis of peripheral pulmonary lesions. Chest 1999; 116: $704-708$

25 Franke KJ, Nilius G, Ruhle $\mathrm{KH}$. Transbronchial catheter aspiration compared to forceps biopsy in the diagnosis of peripheral lung cancer. Eur J Med Res 2009; 14: 13-17

26 Ost $D$, Shah R, Anasco E et al. A randomized trial of CT fluoroscopicguided bronchoscopy vs conventional bronchoscopy in patients with suspected lung cancer. Chest 2008; 134: 507-513

27 Hautmann $H$, Henke MO, Bitterling $H$. High diagnostic yield from transbronchial biopsy of solitary pulmonary nodules using low-dose CTguidance. Respirology 2010; 15: 677-682

28 Herth F, Becker HD, LoCicero J et al. Endobronchial ultrasound in therapeutic bronchoscopy. Eur Respir J 2002; 20: 118-121

29 Kurimoto N, Miyazawa T, Okimasa S et al. Endobronchial ultrasonography using a guide sheath increases the ability to diagnose peripheral pulmonary lesions endoscopically. Chest 2004; 126: 959-965

30 Herth FJ, Eberhardt R, Becker HD et al. Endobronchial ultrasound-guided transbronchial lung biopsy in fluoroscopically invisible solitary pulmonary nodules: a prospective trial. Chest 2006; 129: 147-150

31 Yamada $N$, Yamazaki K, Kurimoto $N$ et al. Factors related to diagnostic yield of transbronchial biopsy using endobronchial ultrasonography with a guide sheath in small peripheral pulmonary lesions. Chest 2007; 132: $603-608$

32 Chao TY, Chien MT, Lie CH et al. Endobronchial ultrasonography-guided transbronchial needle aspiration increases the diagnostic yield of peripheral pulmonary lesions: a randomized trial. Chest 2009; 136: 229236
33 Steinfort DP, Khor YH, Manser RL et al. Radial probe endobronchial ultrasound for the diagnosis of peripheral lung cancer: systematic review and meta-analysis. Eur Respir J 2010; 37: 902 - 910

34 Eberhardt R, Morgan RK, Ernst A et al. Comparison of suction catheter versus forceps biopsy for sampling of solitary pulmonary nodules guided by electromagnetic navigational bronchoscopy. Respiration 2010; 79: 54-60

35 Eberhardt $R$, Anantham $D$, Herth $F$ et al. Electromagnetic navigation diagnostic bronchoscopy in peripheral lung lesions. Chest 2007; 131: $1800-1805$

36 Gildea TR, Mazzone PJ, Karnak D et al. Electromagnetic navigation diagnostic bronchoscopy: a prospective study. Am J Respir Crit Care Med 2006; 174: 982 - 989

37 Makris D, Scherpereel A, Leroy $S$ et al. Electromagnetic navigation diagnostic bronchoscopy for small peripheral lung lesions. Eur Respir J 2007; 29: $1187-1192$

38 Eberhardt R, Anantham D, Ernst $A$ et al. Multimodality bronchoscopic diagnosis of peripheral lung lesions: a randomized controlled trial. Am J Respir Crit Care Med 2007; 176: 36-41

39 Eberhardt R, Kahn N, Gompelmann $D$ et al. LungPoint-a new approach to peripheral lesions. J Thorac Oncol 2010; 5: 1559-1563

40 Asahina $H$, Yamazaki K, Onodera $Y$ et al. Transbronchial biopsy using endobronchial ultrasonography with a guide sheath and virtual bronchoscopic navigation. Chest 2005; 128: $1761-1765$

41 Babiak A, Hetzel J, Krishna G et al. Transbronchial cryobiopsy: a new tool for lung biopsies. Respiration 2009; 78: 203-208

42 Anantham D, Feller-Kopman D, Shanmugham $L N$ et al. Electromagnetic navigation bronchoscopy-guided fiducial placement for robotic stereotactic radiosurgery of lung tumors: a feasibility study. Chest 2007; 132: 930-935

43 Schroeder C, Hejal R, Linden PA. Coil spring fiducial markers placed safely using navigation bronchoscopy in inoperable patients allows accurate delivery of CyberKnife stereotactic radiosurgery. J Thorac Cardiovasc Surg 2010; 140: 1137-1142

44 Harley DP, Krimsky WS, Sarkar S et al. Fiducial marker placement using endobronchial ultrasound and navigational bronchoscopy for stereotactic radiosurgery: an alternative strategy. Ann Thorac Surg 2010; 89: 368 - 373 discussion 373-364

45 Harms $W$, Krempien $R$, Grehn $C$ et al. Electromagnetically navigated brachytherapy as a new treatment option for peripheral pulmonary tumors. Strahlenther Onkol 2006; 182: 108 -111

46 Lencioni $R$, Crocetti L, Cioni R et al. Response to radiofrequency ablation of pulmonary tumours: a prospective, intention-to-treat, multicentre clinical trial (the RAPTURE study). Lancet Oncol 2008; 9: 621 - 628

47 Tanabe T, Koizumi T, Tsushima K et al. Comparative study of three different catheters for CT imaging-bronchoscopy-guided radiofrequency ablation as a potential and novel interventional therapy for lung cancer. Chest 2010; 137: 890-897

48 Tsushima K, Koizumi T, Tanabe T et al. Bronchoscopy-guided radiofrequency ablation as a potential novel therapeutic tool. Eur Respir J 2007; 29: $1193-1200$ 